PROCEEDINGS OF THE

AMERICAN MATHEMATICAL SOCIETY

Volume 132, Number 4, Pages 1087-1090

S 0002-9939(03)07277-0

Article electronically published on October 9, 2003

\title{
ON THE UNIQUENESS OF THE ERGODIC MAXIMAL FUNCTION
}

ROGER L. JONES

(Communicated by Michael Handel)

\begin{abstract}
We give a new (shorter) proof of a result of L. Ephremidze showing that if two functions have the same ergodic maximal function, then they are equal a.e.
\end{abstract}

Let $(X, \Sigma, m)$ denote a complete non-atomic probability space, and let $\tau: X \rightarrow$ $X$ be a measurable ergodic measure-preserving point transformation. Let $f^{\star}(x)=$ $\sup _{n>0} \frac{1}{n} \sum_{k=0}^{n-1} f\left(\tau^{k} x\right)$. In [2] the following result is proven.

Theorem 0.1. Assume $f$ and $g$ are two $L^{1}$ functions such that $f^{\star}(x)=g^{\star}(x)$ for a.e. $x$, then $f=g$ a.e.

In this note we give a different proof of this fact based on the Kakutani skyscraper construction. See [3] where similar ideas are used to study the integrability of the ergodic maximal function.

The following lemma will play a key role in the proof.

Lemma 0.2. Let $\tau$ be a measurable invertible ergodic measure-preserving transformation from $X$ to itself. Assume $f$ and $g$ are in $L^{1}(X)$ with $f^{\star}(x)=g^{\star}(x)$ for a.e. $x$. If $0<m\left\{x: f^{\star}(x) \leq \lambda\right\} \leq 1$, then $f=g$ a.e. on $\left\{f^{\star}>\lambda\right\}$.

Proof. To make the computations below easier, let $F_{\lambda}=f-\lambda$ and $G_{\lambda}=g-\lambda$. Note that we have $F_{\lambda}^{\star}(x)=G_{\lambda}^{\star}(x)$ for a.e. $x$. If we establish that $F_{\lambda}=G_{\lambda}$ on $\left\{F_{\lambda}^{\star}>0\right\}$, then we will have $f=g$ on $\left\{f^{\star}>\lambda\right\}$.

Let

$$
B=\left\{x: F_{\lambda}^{\star}(x) \leq 0\right\}
$$

be the base for the Kakutani skyscraper construction and denote by $C_{1}, C_{2}, \ldots$ the resulting sequence of disjoint columns whose union is the whole space. Let $B_{n}$ denote the base of the $n$th column. Thus $B_{n} \subset B$, the sets $\tau^{i} B_{n}$ are disjoint for $i=0,1, \ldots, n-1$, and $\tau^{n} B_{n} \subset B$.

We claim that for points in the column $C_{n} \backslash B_{n}$, with $n>1$, we have $F_{\lambda}(x)=$ $G_{\lambda}(x)$. To see this we first show that for $x \in C_{n} \backslash B_{n}$ the maximum must occur before leaving the tower. Assume $x \in \tau^{n-i} B_{n}$ for some $i \in\{1,2, \ldots, n-1\}$. Given

Received by the editors November 28, 2002.

2000 Mathematics Subject Classification. Primary 28D05, 37A05.

The author is partially supported by a research leave granted by DePaul University's Research Council.

(C)2003 American Mathematical Society 
$\epsilon>0$, there is a positive integer $m$ such that

$$
0<F_{\lambda}^{\star}(x)<\frac{1}{m} \sum_{k=0}^{m-1} F_{\lambda}\left(\tau^{k} x\right)+\epsilon .
$$

Take $\epsilon<\frac{1}{i+1} F_{\lambda}^{\star}(x)$. If $m>i$, then using the fact that $\tau^{i} x \in B$, and for $y \in B$ we know $\sum_{k=0}^{\ell} F_{\lambda}\left(\tau^{k} y\right) \leq 0$, we have

$$
0<F_{\lambda}^{\star}(x)<\frac{1}{m}\left(\sum_{k=0}^{i-1} F_{\lambda}\left(\tau^{k} x\right)+\sum_{k=i}^{m-1} F_{\lambda}\left(\tau^{k} x\right)\right)+\epsilon \leq \frac{1}{m} \sum_{k=0}^{i-1} F_{\lambda}\left(\tau^{k} x\right)+\epsilon .
$$

Because of our choice of $\epsilon$ we now have

$$
0<F_{\lambda}^{\star}(x)<\frac{1}{m} \sum_{k=0}^{i-1} F_{\lambda}\left(\tau^{k} x\right)+\epsilon<\frac{i}{m} F_{\lambda}^{\star}(x)+\frac{1}{i+1} F_{\lambda}^{\star}(x) \leq F_{\lambda}^{\star}(x) .
$$

Since we cannot have $F_{\lambda}^{\star}(x)<F_{\lambda}^{\star}(x)$ we must have $m \leq i$.

If $x \in \tau^{n-1} B_{n}$, the top level of the column, we see that $F_{\lambda}^{\star}(x)=F_{\lambda}(x)$ and $G_{\lambda}^{\star}(x)=G_{\lambda}(x)$, since the maximum must occur in the tower and we are already at the top of the tower. Thus $F_{\lambda}(x)=G_{\lambda}(x)$.

Now we argue by induction on the distance from the top of the tower. Assume we are in the set $\tau^{n-i} B_{n}$, that is, there are $i-1$ levels above our current level. By induction, we can assume $F_{\lambda}(x)=G_{\lambda}(x)$ for $x \in \bigcup_{j=1}^{i-1} \tau^{n-j} B_{n}$. Since the maximum must occur while we are still in the tower, and $F_{\lambda}=G_{\lambda}$ for all $x$ above the current level, if $F_{\lambda}(x) \neq G_{\lambda}(x)$ for $x$ in the current level, we could not have $F_{\lambda}^{\star}(x)=G_{\lambda}^{\star}(x)$. Thus we have $F_{\lambda}=G_{\lambda}$ on $\left\{F_{\lambda}^{\star}>0\right\}$ or $f=g$ on $\{f>\lambda\}$.

The following lemma (and proof) appears in 2]. We include it here for completeness. In what follows let $I(f)$ denote the essential infinum of $f^{\star}$ and $E(f)=$ $\int_{X} f(x) d m(x)$.

Lemma 0.3. Let $\tau$ be a measurable invertible ergodic measure-preserving transformation from $X$ to itself. Then $I(f)=\int_{X} f(x) d m(x)$.

Proof. By the ergodic theorem, $f^{\star}(x) \geq E(f)$ a.e. since for a.e. $x$ we know that $\lim _{n \rightarrow \infty} \frac{1}{n} \sum_{k=0}^{n-1} f\left(\tau^{k} x\right)=E(f)$. Thus $I(f)=E(f)+\alpha$ for some $\alpha \geq 0$. The maximal ergodic theorem says that for $\lambda \in \mathbb{R}$ we have $\lambda m\left\{f^{\star}>\lambda\right\} \leq \int_{\left\{f^{\star}>\lambda\right\}} f(x) d m(x)$. Let $\lambda=E(f)+\frac{\alpha}{2}$. Then the left-hand side is $E(f)+\frac{\alpha}{2}$ while the right-hand side is $E(f)$. Hence $E(f)+\frac{\alpha}{2} \leq E(f)$, or $\alpha=0$.

We now complete the proof in the case that $\tau$ is invertible. We have three possible cases.

Case 1. Assume $m\left\{x: f^{\star}(x)=I(f)\right\}=0$. In this case we can select a decreasing sequence $\left\{\lambda_{1}, \lambda_{2}, \ldots\right\}$ such that $\lim _{n \rightarrow \infty} \lambda_{n}=I(f)$. By Lemma 0.3 each of the sets $\left\{f^{\star}>\lambda_{n}\right\}$ has positive measure, and hence we can apply Lemma 0.2. Applying Lemma 0.2, we see that for each $\lambda_{n}$ we have $f(x)=g(x)$ for a.e. $x \in\left\{f^{\star}>\lambda_{n}\right\}$. Since the union of these sets is $\left\{f^{\star}>I(f)\right\}$, a set of full measure, we are done.

Case 2. Assume $0<m\left\{x: f^{\star}(x)=I(f)\right\}<1$. In this case, with $\lambda=I(f)$ we already have by Lemma 0.2 that $f(x)=g(x)$ for a.e. $x$ in $\left\{f^{\star}>I(f)\right\}$. Using the notation in the proof of Lemma 0.2 we will show that for a.e. 
$x \in B_{n}$, we have $\sum_{k=0}^{n-1} F_{\lambda}\left(\tau^{k} x\right)=0$ (and the same for $G_{\lambda}$ ). Let $H_{n}=$ $\left\{x \in B_{n}: \sum_{k=0}^{n-1} F_{\lambda}\left(\tau^{k} x\right)<0\right\}$. If $m\left(H_{n}\right) \neq 0$ for some $n$, then using the fact that for $x \in B_{n}$ we know $\sum_{k=0}^{n-1} F_{\lambda}\left(\tau^{k} x\right) \leq 0$, we have

$$
\begin{aligned}
0 & =\int_{X} F_{\lambda}(x) d m(x) \\
& =\sum_{n=1}^{\infty} \int_{B_{n} \backslash H_{n}} \sum_{k=0}^{n-1} F_{\lambda}\left(\tau^{k} x\right) d m(x)+\sum_{n=1}^{\infty} \int_{H_{n}} \sum_{k=0}^{n-1} F_{\lambda}\left(\tau^{k} x\right) d m(x) \\
& =\sum_{n=1}^{\infty} \int_{H_{n}} \sum_{k=o}^{n-1} F_{\lambda}\left(\tau^{k} x\right) d m(x)<0
\end{aligned}
$$

We cannot have $0<0$; thus we must have $m\left(H_{n}\right)=0$ for each $n$.

Since we have $F_{\lambda}\left(\tau^{k} x\right)=G_{\lambda}\left(\tau^{k} x\right)$ for $k=1,2, \ldots, n-1$, we must have $F_{\lambda}(x)=G_{\lambda}(x)$ too.

Case 3. Assume $m\left\{x: f^{\star}(x)=I(f)\right\}=1$. In this case, since $I(f)=I(g)$, we have by Lemma 0.3 that their integrals are the same and both equal $I(f)$. Since our assumption then implies that $f$ (and $g$ ) cannot be more than their integral, both $f$ and $g$ must be constant a.e.

To handle the non-invertible case, form the natural extension of $(X, \Sigma, m, \tau)$ (see [1], p. 240) resulting in a new space $(\tilde{X}, \tilde{\Sigma}, \tilde{m}, \tilde{\tau})$ with $\tilde{\tau}$ invertible. Let $\tilde{f}\left(x_{0}, x_{1}, \ldots\right)=f\left(x_{0}\right)$ and $\tilde{g}\left(x_{0}, x_{1}, \ldots\right)=g\left(x_{0}\right)$. It is clear that if $f^{\star}(x)=g^{\star}(x)$, then $\tilde{f}^{\star}(x)=\tilde{g}^{\star}(x)$, and hence we must have $\tilde{f}=\tilde{g}$ a.e. This implies that $f=g$ in the non-invertible case too.

Remark 0.4. In 22 Ephremidze shows that the analogue of Theorem 0.1 is false if $m(X)=\infty$. However, in the case $m(X)=\infty$, he proves that $f^{\star}=g^{\star}$ a.e. implies $f=g$ a.e. on $\left\{f^{\star}>0\right\}$. Using this he shows that the conclusion of Theorem 0.1 holds if $f$ and $g$ are nonnegative $L^{1}$ functions.

Remark 0.5. The proof in [2] involves first proving results for the discrete maximal operator on $\mathbb{Z}$ and using these results to obtain conclusions in the dynamical system. In the above argument we work directly in the dynamical system. This allows us to take advantage of the recurrence properties and the fact that we have a finite measure space.

\section{ACKNOWLEDGMENT}

The above argument benefitted from discussions with Isaac Kornfeld, Joseph Rosenblatt and Ayşe Şahin. Furthermore, the referee made suggestions which greatly improved the presentation and completeness of the paper.

\section{REFERENCES}

[1] I. P. Cornfeld, S. V. Fomin, and Y. G. Sinai, Ergodic Theory, Springer-Verlag, New York, 1982. MR 87f:28019

[2] L. Ephremidze, On the uniqueness of the ergodic maximal function, Fundamenta Mathematicae 174 (2002) 217-228. MR 2003e:37003] 
[3] R. L. Jones Inequalities for the ergodic maximal function, Studia Math. 40 (1977) 111-129. MR 55:3215

Department of Mathematics, DePaul University, 2330 N. Kenmore, Chicago, Illinois 60614

E-mail address: rjones@condor.depaul.edu 